\title{
C-Reactive protein reactions to glucose- insulin-potassium infusion and relations to infarct size in patients with acute coronary syndromes
}

Hadeel Alkofide ${ }^{1,2}$, Gordon S. Huggins ${ }^{3}$, Joni R. Beshansky ${ }^{4,5}$, Robin Ruthazer ${ }^{4,6}$, Inga Peter ${ }^{7}$, Madhab Ray ${ }^{1}$, Jayanta T. Mukherjee ${ }^{1,6}$ and Harry P. Selker ${ }^{4,6^{*}}$

\begin{abstract}
Background: Some benefits of glucose-insulin-potassium (GIK) in patients with acute coronary syndromes (ACS) may be from an anti-inflammatory effect. The primary aim of this study was to assess the impact of GIK administration early in the course of ACS on inflammatory marker C-reactive protein (CRP) levels. A secondary aim was to investigate the association between CRP and 30-day infarct size.

Methods and Results: Retrospective analysis of participants with ACS randomly assigned to GIK or placebo for at least $8 \mathrm{~h}$ in the IMMEDIATE Trial biological mechanism cohort $(n=143)$. High sensitivity CRP (hs-CRP) was measured at emergency department presentation, and 6 and $12 \mathrm{~h}$ into infusion. Logarithmically transformed hs-CRP values at 12-hours were lower with GIK vs. placebo (mean $=0.65 \mathrm{mg} / \mathrm{L}$ in $\mathrm{GIK}, 0.84 \mathrm{mg} / \mathrm{L}$ in placebo), with a marginal trend toward significance $(P=0.053)$. Furthermore, using mixed models of hs-CRP, time, and study group, there was a significant increase in hs-CRP levels over time, but the rate of change did not differ between treatment arms $(P=0.3)$. Multivariable analysis showed that an elevation in hs-CRP, measured at $12 \mathrm{~h}$, was an independent predictor of 30-day infarct size ( $\beta$ coefficient, 6.80; $P=0.04$ ) using sestamibi SPECT imaging.
\end{abstract}

Conclusions: The results of this study show no significant effect of GIK on hs-CRP. In addition our results show that in patients with ACS, hs-CRP measured as early as $12 \mathrm{~h}$ can predict 30-day infarct size.

Keywords: Acute coronary syndromes, Glucose-insulin-potassium (GIK), Inflammation, C-reactive protein, Metabolic therapy

\section{Backgrounds}

Glucose -insulin-potassium (GIK) infusion as metabolic therapy can reduce damage to myocardial cells in the setting of ischemia or infarction [1-4]. Since its introduction in the early 1960s [5], GIK treatment has been assessed in both animal models and human studies. However, the role of GIK in patients with acute myocardial infarction (MI) remains controversial. While several clinical trials have shown no benefit of GIK treatment in

\footnotetext{
* Correspondence: hselker@tuftsmedicalcenter.org

${ }^{4}$ Center for Cardiovascular Health Services Research, Institute for Clinical Research and Health Policy Studies, Tufts Medical Center, Boston, MA, USA ${ }^{6}$ Tufts Clinical and Translational Science Institute, Tufts University, Boston, MA, USA

Full list of author information is available at the end of the article
}

patients with acute MI [6-10], other studies have reported benefit [11-15]. Most recently, the IMMEDIATE (Immediate Myocardial Metabolic Enhancement During Initial Assessment and Treatment in Emergency care) Trial failed to show significant differences in the outcome of progression to $\mathrm{MI}$ among patients presenting with acute coronary syndromes (ACS); but it did show that GIK was associated with lower rates of the composite outcome of cardiac arrest and/or in-hospital mortality, and with smaller infarct size [15]. The IMMEDIATE Trial is distinguished from other trials by the early administration of GIK prior to arrival at the hospital [15]. In previous studies, GIK administration was delayed until hospital admission [6-10], with a median time of 
$6 \mathrm{~h}$ from symptom onset to GIK initiation in one study [9].

While the mechanisms underlying GIK's effects in patients with ACS are unknown, several pathways have been suggested, including a metabolic pathway by promoting of glycolysis, and reducing circulating free fatty acid (FFA) levels, and through antiinflammatory effects [16-18]. Inflammation contributes to myocardial damage in ischemia, infarction, and reperfusion [19]. The acute phase reactant Creactive protein (CRP) is released in response to inflammation in chronic coronary artery disease and in acute MI [19]. High CRP levels after acute MI predict infarct expansion and plaque rupture [20-22]. Whereas a reduction in the rise of CRP levels has been shown to indicate the efficacy of thrombolytic therapy and a patent infarct-related coronary artery $[23,24]$. Continued elevations in CRP portend increased risk of mortality, even in the presence of currently available therapies for ACS [25].

In the setting of ACS, GIK has been demonstrated to reduce serum markers of inflammation, including CRP in one study [18] but not in others [26, 27]. Here, in the largest study to date of very early administration of GIK for ACS, the IMMEDIATE Trial, we tested the hypothesis that GIK was associated with smaller CRP elevations compared to those treated with placebo. Related to this, we further hypothesized that the changes in CRP levels following presentation with an ACS correlate with 30day infarct size.

\section{Methods}

\section{Study sample}

This study analyzed data collected from participants enrolled in the IMMEDIATE Trial, the methodology of which has been published elsewhere [15]. In brief, it was a randomized, placebo-controlled, double-blind clinical effectiveness trial of GIK, from December 2006 through July 2011, in which paramedics, aided by electrocardiograph (ECG)-based decision support, randomized and enrolled 871 participates aged $\geq 30$ years with high probability of ACS [15]. Participants were given either the GIK solution (30\% glucose, $50 \mathrm{U} / \mathrm{L}$ of regular insulin, and $80 \mathrm{mEq}$ of $\mathrm{KCl} / \mathrm{L}$ ) intravenously at $1.5 \mathrm{~mL} / \mathrm{kg} / \mathrm{h}$ for $12 \mathrm{~h}$, or an identical-appearing placebo as $5 \%$ glucose solution. In the study, the median time from symptom onset to initiation of infusion was $90 \mathrm{~min}$ [15]. This investigation was based on the IMMEDIATE Trial biological mechanism cohort ("biocohort"), which comprised of participants who consented to participate in this biocohort, confirmed as having ACS, and treated for at least $8 \mathrm{~h}$ with study drug. Confirmed ACS (acute MI or angina pectoris) was determined by site investigator review and then independently adjudicated by the study clinical events committee who were blinded to study group, glucose, and potassium results. Enrolment in the biocohort began after the trial was started and only included 6 out of 13 study centers. The study protocol and analyses were approved by the Tufts Institutional Review Board (IRB) and by the IRBs responsible for each of the IMMEDIATAE Trial study sites.

\section{Data collection}

During the 12-hour infusion of study drug, glucose, insulin, FFA, and high sensitivity CRP (hs-CRP) levels were tested at three times: 1) the initial measurement, which was drawn as soon as feasible after hospital arrival; 2) $6 \mathrm{~h}$ after start of study drug; and 3) $12 \mathrm{~h}$ after start of study drug, or upon discontinuation of the infusion. Hemoglobin $\mathrm{A} 1 \mathrm{C}$ was measured on admission and at 30 days, and infarct size was measured at 30 days by sestamibi SPECT imaging. The Trial's core laboratory interpreted the nuclear studies, and the core laboratory of Tufts Clinical and Translational Science Institute performed the hs-CRP measurements. Other covariates measured in the biocohort include demographic data, vital signs, medical history, and medications used at home, in the hospital, or upon discharge.

\section{Data analysis}

Statistical analyses were performed using $\mathrm{R}$, version 2.15.2. Descriptive statistics were used to describe baseline characteristics. All tests were two-sided, using alpha $<0.05$ to determine statistical significance. Serum hsCRP levels ranged widely and their distribution was highly skewed to lower levels; therefore hs-CRP concentrations were logarithmically (base 10) transformed in all further analyses. Linear regression models were used to assess the relationship between initial hs-CRP measurements and baseline characteristics; variables significantly associated with initial hs-CRP levels were adjusted-for in the analysis. The independent sample t-test was used to detect unadjusted differences in cross-sectional hs-CRP levels between GIK and placebo. In addition, using independent sample $t$-test, the differences between the initial and 12-hour hs-CRP measurements (delta hs-CRP) were used to assess differences by treatment arm. A mixed model was used to detect the differential effect of treatment on the rate of change in the initial, 6-, and 12hour hs-CRP levels, adjusting for baseline and clinical characteristics associated with initial hs-CRP measurement, and accounting for repeated measures on each participant at the different time points. We also adjusted for time from symptom onset to reperfusion therapy. The linear mixed model uses a random intercept for each subject, and for within-subject correlation. The model included terms for time, from the initiation of infusion (real-time as a continuous measurement), and an 
interaction term between study drug and time, in order to estimate the association between treatment arms and change in the inflammatory marker over the follow-up time points. Sensitivity analysis was done by stratifying participants based on ACS diagnosis (i.e. acute MI compared to unstable angina), and by the use of statins prior to enrollment in the study.

Univariate and multivariable linear regression models were used to study the association between hs-CRP levels at the three time points (as the predictor) and infarct size (as the outcome), after adjustment for potential confounders including age, gender, and those correlated with infarct size in univariate models at a $p$-value of $<0.1$. Infarct sizes ranged from 0 to $59 \%$ of left ventricular mass, where $0 \%$ represent those who did not develop an infarct, and $59 \%$ was assigned to those who died $(n=4)$ before imaging, based on the fact that the largest infarct size measured in the study was $58 \%$. In addition, correlations between hs-CRP at the initial, 6-, and 12-hour determinations and infarct size were assessed using Spearman's rank correlation. Sensitivity analyses were performed by removing participants who had no infarct to check if the association between hsCRP and infarct size has the same direction for those who had an infarct versus those who did not. Additional sensitivity analysis was done on participants in the placebo group only to test for the correlation between hs-CRP levels, at the three time points measured, and infarct size, without the effect of GIK. We also ran sensitivity analyses based on ACS diagnosis, and prior use of statins. Because hs-CRP baseline levels were not measured prior to study infusion initiation, but rather, after hospital arrival, the time from when the drug started and time of the first hs-CRP measurement was used as a covariate in regression models.

\section{Results}

\section{Characteristics of study population}

A total of 143 participants met the inclusion criteria for the biocohort; 68 received GIK and 75 placebo. Not all individuals had complete hs-CRP measurements; participants with at least one hs-CRP measurement available were included in the analysis $(n=143)$. Additional file 1 : Figure S1 illustrates how the biocohort participants were enrolled. Their demographic and clinical characteristics by treatment arm are shown in Table 1 . The average age was 64 years in both groups; $77 \%$ of the GIK group and $70 \%$ of the placebo group were men. Chest pain was the chief symptom of $86 \%$ of participants, and they were randomized at a median time of $90 \mathrm{~min}$ after ischemic symptom onset. The median time from start of study drug to the measurement of the initial hs-CRP values was $2.5 \mathrm{~h}$ in the GIK group and $2.6 \mathrm{~h}$ in the placebo group. The entry participant characteristics were well- balanced in the GIK and placebo groups. The clinical characteristics of participants in the biocohort were similar to all IMMEDIATE Trial participants by site of enrollment. The diagnosis of ACS was more common in the biocohort, as expected because a confirmed diagnosis of ACS was a requirement for enrollment into the biocohort (Additional file 1: Table S1).

\section{Changes in hs-CRP levels}

Table 2 shows hs-CRP levels in the first $12 \mathrm{~h}$ of drug infusions before logarithmic transformation. Linear regression models were performed to assess the association between admission hs-CRP levels and baseline characteristics. They showed that older people, women and individuals with history of heart failure had higher hs-CRP levels upon admission (Additional file 1: Table S2). In addition the longer time from symptom onset to reperfusion therapy the higher the hs-CRP levels (Additional file 1: Table S2). These associations remained significant after adjusting for the use of study drug. No other clinical characteristics had a significant association with admission hs-CRP values.

The hs-CRP measurements increased significantly in both the control and the treatment groups by 6 and $12 \mathrm{~h}$, compared with the initial measurement $(P<0.01$ for all intragroup comparisons). Hs-CRP values were not different for those who received GIK versus placebo at the initial and 6-hour measurements; however by $12 \mathrm{~h}$, the hs-CRP levels were slightly higher in placebo-treated versus GIKtreated participants $(P=0.053)$ (Fig. 1). When comparing delta hs-CRP, the differences between the initial and 12hour hs-CRP measurements, there were no significant differences in those delta values between treatment arms (Additional file 1: Table S3). Using linear mixed model, hsCRP levels increased significantly with time in both arms $(P<0.01)$; however, the rate of change did not differ between the GIK and placebo groups $(P=0.30$ for time"treatment interaction). Baseline characteristics associated with initial hs-CRP were added to the model and did not change the above results. In addition, the results remained the same after adjusting for the time from symptom onset to reperfusion therapy. The results also did not change when we stratified the analysis by ACS diagnosis, and by the use of statin therapy prior to study enrollment.

\section{Association between hs-CRP and infarct size}

After adjusting for age, gender and GIK administration (variables that were associated with infarct size in univariate models), there were no significant associations between hs-CRP and infarct size at the initial and 6-hour measurements. On the other hand the 12-hour measurement and delta hs-CRP were significantly associated with infarct size (Table 3). Sensitivity analysis done by removing participants with no infarct (i.e. those with an infarct 
Table 1 Baseline demographics and clinical characteristics of study participants by treatment group in the biocohort $(N=143)^{a}$

\begin{tabular}{|c|c|c|}
\hline \multirow[t]{3}{*}{ Cohort characteristics } & \multicolumn{2}{|l|}{ No. (\%) } \\
\hline & \multicolumn{2}{|l|}{$N=143$} \\
\hline & $\mathrm{GIK}(N=68)$ & Placebo $(N=75)$ \\
\hline Age, mean (SD), y & $64.5(12.9)$ & $63.9(12.8)$ \\
\hline Men & $52(76.5)$ & $52(69.3)$ \\
\hline \multicolumn{3}{|l|}{ Race } \\
\hline White & $66(97)$ & $71(95)$ \\
\hline Hispanic ethnicity & $3(4.4)$ & $7(9.3)$ \\
\hline \multicolumn{3}{|l|}{ Chief complaint on presentation } \\
\hline Chest pain & $59(86.8)$ & $67(89.3)$ \\
\hline Shortness of breath & $1(1.5)$ & $3(4)$ \\
\hline Other ${ }^{b}$ & $8(11.7)$ & $5(6.7)$ \\
\hline \multicolumn{3}{|l|}{ Medical history } \\
\hline Diabetes & $12(17.6)$ & $19(25.3)$ \\
\hline Heart Failure & $4(5.9)$ & $7(9.3)$ \\
\hline AMl & $21(30.9)$ & $24(32.0)$ \\
\hline \multicolumn{3}{|l|}{ Medication history } \\
\hline Statins & $30(44.1)$ & $29(38.7)$ \\
\hline Aspirin & $39(57.3)$ & $44(58.7)$ \\
\hline Minutes from symptom onset to study drug, median (IQR) & $86(51.5-160.5)$ & $81(53-123)$ \\
\hline \multicolumn{3}{|l|}{ Minutes from symptom onset to study drug } \\
\hline $0-30$ & $1(1.5)$ & $0(0)$ \\
\hline $31-60$ & $21(30.9)$ & $22(29.3)$ \\
\hline $61-90$ & $9(13.2)$ & $19(25.3)$ \\
\hline $91-180$ & $12(17.7)$ & $15(20.0)$ \\
\hline $181-360$ & $10(14.7)$ & $8(10.7)$ \\
\hline $361-24 \mathrm{~h}$ & $6(8.8)$ & $5(6.7)$ \\
\hline Within 24 h, unspecified & $3(4.4)$ & $4(5.3)$ \\
\hline$>24 \mathrm{~h}$ & $6(8.8)$ & $2(2.7)$ \\
\hline ACl-TIPI score, mean (SD), \% & $83(15.7)$ & $83.1(12.1)$ \\
\hline \multicolumn{3}{|l|}{ Hospital reperfusion treatment } \\
\hline Thrombolytic therapy & $1(1.5)$ & $1(1.3)$ \\
\hline $\mathrm{PCl}$ & $59(86.8)$ & $56(74.7)$ \\
\hline Coronary artery bypass graft & $0(0)$ & $2(2.7)$ \\
\hline \multicolumn{3}{|l|}{ Confirmed diagnosis } \\
\hline Acute myocardial infarction & $58(85.3)$ & $68(90.7)$ \\
\hline Any angina & $10(14.7)$ & $7(9.3)$ \\
\hline \multicolumn{3}{|c|}{ Time from study drug to biomarker measurement, median (IQR), hours } \\
\hline Initial & $2.5(1.3-3.3)$ & $2.6(1.9-3.2)$ \\
\hline $6 \mathrm{~h}$ & $6(6-6.3)$ & $6(6-6.2)$ \\
\hline $12 \mathrm{~h}$ & $12(12-12.2)$ & $12.1(12-12.3)$ \\
\hline
\end{tabular}

$A M I$ indicates acute myocardial infarction; $A C I-T I P I$ acute cardiac ischemia time-insensitive predictive instrument; GIK glucose-insulin-potassium; IQR interquartile range; $P C l$ percutaneous coronary intervention; $S D$ standard deviation

${ }^{a}$ No significant differences were noted between GIK and placebo groups

${ }^{\mathrm{b}}$ Abdominal pain, back pain, dizziness, heartburn, loss of consciousness, shoulder pain and weakness 
Table 2 Hs-CRP Levels in the first 12 h of emergency department admission

\begin{tabular}{|c|c|c|c|c|c|c|}
\hline \multirow[b]{2}{*}{$\mathrm{Hs}-\mathrm{CRP}$} & \multicolumn{3}{|l|}{ GIK } & \multicolumn{3}{|c|}{ Placebo } \\
\hline & No. & Mean (SD) & Median (IQR) & No. & Mean (SD) & Median (IQR) \\
\hline Initial hs-CRP mg/L & 59 & $8.1(17.7)$ & $3.1(1.4-7.3)$ & 61 & $12.3(24.4)$ & $3.2(1.9-9.3)$ \\
\hline $6 \mathrm{~h} \mathrm{hs-CRP} \mathrm{mg/L}$ & 58 & $8.6(17.0)$ & $3.4(1.8-8.8)$ & 63 & $15.1(30.7)$ & $4.2(2.0-12.4)$ \\
\hline $12 \mathrm{~h}$ hs-CRP mg/L & 57 & $9.2(16.7)$ & $4.5(2.3-7.9)$ & 64 & $17.6(31.0)$ & $5.9(3.1-13.7)$ \\
\hline
\end{tabular}

GIK indicates glucose-insulin-potassium; $\mathrm{Hs}$-CRP high sensitivity C-reactive protein; IQR interquartile range; $S D$ standard deviation

size of 0 , or those who died and did not have infarct size measurement available) $(n=31)$ showed the same association between hs-CRP levels and infarct size (Table 3). Also stratifying participants by ACS diagnosis and statin use prior to study enrollment did not significantly change the study results. Results from spearman's rank correlation yielded similar associations between hs-CRP and 30-day infarct size (Additional file 1: Fig. S2). When testing for the correlation between hs-CRP and infarct size in the placebo group only, we found that the 12-hour hs-CRP and the delta hs-CRP remained significantly correlated with infarct size ( $p$-value $=0.022$ and 0.049 , for the $12 \mathrm{~h}$ and delta hs-CRP respectively).

\section{Discussion}

Our data confirm that plasma hs-CRP concentrations are increased in participants presenting to the ED with ACS, presumably reflecting a state of inflammation. However, the administration of GIK early in ACS did not have a significant impact on hs-CRP levels. Although there was a modest difference between the 12-hour hs-CRP levels with GIK, the delta hs-CRP values was not different between treatment arms. In addition, results of mixed models, adjusting for within and between subjects variability, demonstrated no effect of GIK on hs-CRP over the three time points. These results suggest that the beneficial effects of GIK in ACS observed in the main study [15], at least as reflected in the absence of an effect on hs-CRP, are less likely to be through an anti-inflammatory effect, and may be more extended through a metabolic effect.

Previous studies on the effect of GIK on CRP yielded conflicting results. In a study by Chaudhuri et al., GIK administration started in the emergency department in patients presenting with ST-segment elevation myocardial infarction (STEMI) $(n=32)$ and lasting $48 \mathrm{~h}$ showed significantly reduced hs-CRP values at 24 and 48 h postinfusion compared to placebo [18]. In contrast, Parikh et al., demonstrated in 25 patients with STEMI that a 24hour infusion of GIK produced no statistically significant difference in $24 \mathrm{~h}$ hs-CRP levels compared with placebo [26]. Additionally a study by Hashemian et al., showed no effect of GIK on hs-CRP levels in 72 patients with STEMI treated within $12 \mathrm{~h}$ from symptom onset [27]. Although those studies added GIK to standard care, there are important differences in the use of GIK in the IMMEDIATE Trial. First, unlike prior clinical trials in which GIK was started typically an average of $6 \mathrm{~h}$ after onset of ischemic symptoms, following documentation of acute MI $[6,10,14]$, in IMMEDIATE, the study drug was started prior to arrival to emergency department, upon emergency medical services (EMS) arrival in the community following a 9-1-1 call, at an average of $90 \mathrm{~min}$ after symptom onset [15]. Moreover, the previous studies only included participants with STEMI. In contrast, the IMMEDIATE Trial included participants with ACS, i.e., either unstable angina or acute MI (whether or not STEMI) [15].

Infarct size has shown to be a prognostic marker of adverse clinical outcomes after an acute coronary event [28]. Baseline CRP levels in healthy individuals or in patients with stable angina are independent risk factor for cardiovascular events [29]. Also the rise in CRP after acute MI or during unstable angina pectoris has been shown to be related to outcome $[23,30,31]$. In this

Table 3 Regression analysis of hs-CRP levels and 30-day infarct size ${ }^{a}$

\begin{tabular}{|c|c|c|c|c|c|c|}
\hline \multirow[b]{2}{*}{ Regression models ${ }^{b}$} & \multicolumn{3}{|c|}{ All participants } & \multicolumn{3}{|c|}{ Participants with an infarct } \\
\hline & No. & Beta Coefficient & $P$-value & No. & Beta Coefficient & P-value \\
\hline Initial hs-CRP mg/L ${ }^{c}$ & 83 & 2.3 & 0.46 & 54 & 3.8 & 0.35 \\
\hline $6 \mathrm{~h} \mathrm{hs-CRP} \mathrm{mg/L^{c }}$ & 85 & 4.0 & 0.17 & 56 & 5.9 & 0.13 \\
\hline $12 \mathrm{~h} \mathrm{hs-CRP} \mathrm{mg/L^{c }}$ & 83 & 6.8 & 0.04 & 56 & 10.6 & 0.02 \\
\hline Delta hs-CRP mg/L ${ }^{d}$ & 78 & 13.9 & 0.02 & 51 & 23.1 & 0.01 \\
\hline
\end{tabular}

GIKGIK indicates glucose-insulin-potassium,: $\mathrm{Hs}$-CRP high sensitivity $\mathrm{C}$-reactive protein

aData analyzed using logarithmically transformed hs-CRP values

${ }^{\mathrm{b}}$ Adjusted for age, gender and GIK administration. In addition, the time from when the drug started to the time of the first hs-CRP measurement was used as a covariate. The coefficient represents the fitted increase in infarct size per one unit change in logarithmically trasnformed hs-CRP.

${ }^{\mathrm{C}}$ Per 1 unit increase in hs-CRP levels

${ }^{\mathrm{d}}$ Difference between the initial hs-CRP and $12 \mathrm{~h}$ hs-CRP measurements 


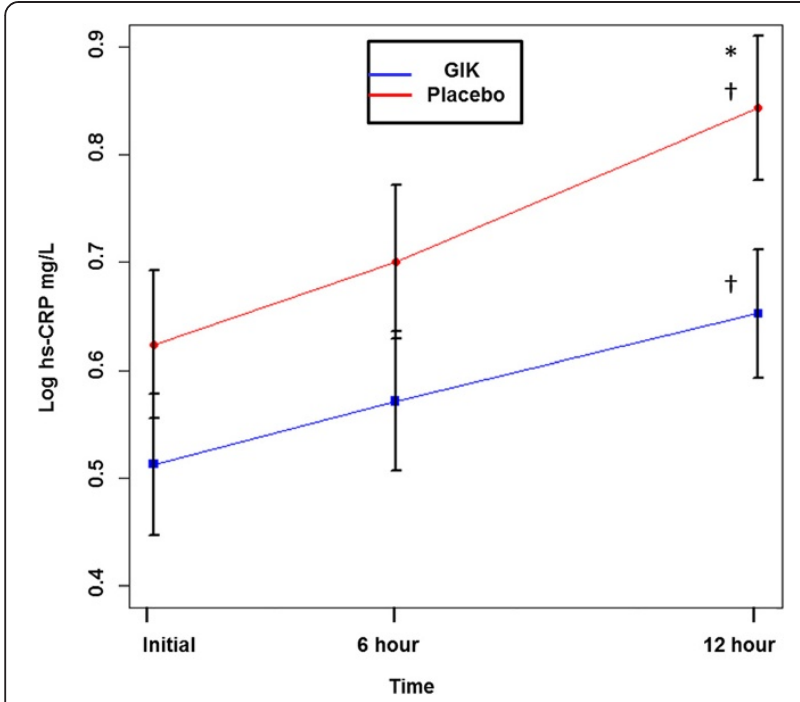

Fig. 1 hs-CRP Levels per Treatment Arm. Time course of mean \pm SEM hs-CRP at initial, $6 \mathrm{~h}$ and $12 \mathrm{~h}$ per treatment arm. GIK indicates glucose-insulin-potassium; $\mathrm{Hs}$-CRP high sensitivity C-reactive protein ${ }^{*} P=0.053$ between groups at $12 \mathrm{~h}$ (independent sample t-test). ${ }^{\dagger}$ $P^{<} 0.01$ within group differences between initial and $6 \mathrm{~h}, 6 \mathrm{~h}$ and $12 \mathrm{~h}$, and initial and $12 \mathrm{~h}$ (paired sample t-test). Initial time represents the first hs-CRP measurement (median $=2.5 \mathrm{~h}$ )

study on participants presenting to EMS with ACS, we document a relationship between hs-CRP level measured at $12 \mathrm{~h}$ and 30-day infarct size. In addition, the magnitude of change in hs-CRP levels, between the initial and 12-hour values, was related to infarct size. Limiting the analysis to those participants with a documented infarct size measurement, and also to those in the placebo group only, showed similar results. Previous studies have shown no clear relationship between CRP levels on hospital admission and infarct size in patients with acute coronary events [32]; but nevertheless, cumulative or peak CRP levels have been correlated with infarct size [33]. Peak CRP levels are reached by no earlier than $24 \mathrm{~h}$ after infarction [34]. Adding to that picture, our hsCRP measurements reflect that an early rise, within $12 \mathrm{~h}$ of ACS symptom onset, correlates with 30-day infarct size. Imaging studies in IMMEDIATE Trial biocohort participants at 30 days showed an $80 \%$ reduction infarct size associated with GIK, both for the entire ACS cohort $(n=110)$ and in those presenting with STEMI $(n=75)$ [15]. Therefore the modest reduction seen in hs-CRP levels at $12 \mathrm{~h}$ between the two groups may be indirectly related to infarct size.

This study has several limitations. First, although our sample is larger than previous studies on the effect of GIK on hs-CRP levels $[18,26,27]$. the size of the IMMEDIATE Trial biocohort may have limited our power to detect treatment interactions. Second, CRP levels prior to the onset of GIK infusion and after the $12 \mathrm{~h}$ infusion were not available. Finally, although CRP is commonly used as an inflammatory biomarker, it is somewhat nonspecific and other biomarkers have been considered as an alternative to CRP. For instance, serum amyloid A, interleukin-6, and adhesion molecules such as soluble intercellular adhesion molecule type 1, similar to CRP, are markers of inflammation that are produced by the liver [35]. Therefore, if GIK exerts an anti-inflammatory effect it may be reflected through biomarkers other than CRP. Nevertheless this study has several strengths including serial hs-CRP levels measurements within $12 \mathrm{~h}$ after GIK initiation, compared to other studies, in which the effect of GIK on CRP was assessed at 24-48 h following treatment. Moreover, our data were collected in a randomized placebo-controlled trial, with both GIK and placebo participants having balanced characteristics.

\section{Conclusions}

In patients with an ACS, early administration of GIK appears to have no significant effect on hs-CRP levels measured in the first $12 \mathrm{~h}$ of treatment infusion. This is consistent with a model that the primary immediate benefits of GIK are more likely metabolic rather than anti-inflammatory. Nevertheless, in order to more fully understand the mechanisms by which GIK exert its potential benefits in patients with an ACS, future research should consider markers of endothelial dysfunction, microvascular dysfunction, and coagulation. Our findings of a possible association between the early rise in hs-CRP levels and infarct size, are consistent with the role of inflammation in extent of infarction, an effect that deserves further investigation as a marker of acute myocardial damage and inflammation.

\section{Additional file}

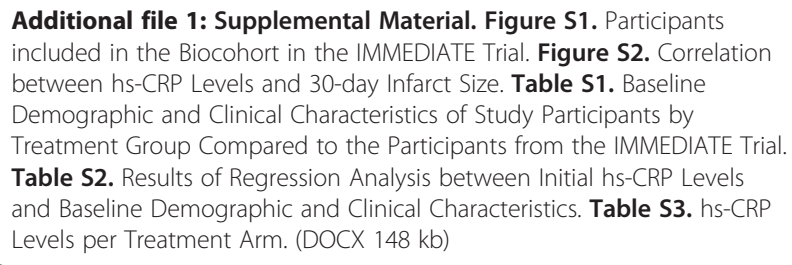

\section{Abbreviations}

ACS: Acute coronary syndromes; CRP: C-reactive protein; EMS: Emergency medical services; FFA: Free fatty acid; GIK: Glucose-insulin-potassium; hsCRP: High sensitivity C-reactive protein; IMMEDIATE: Immediate Myocardial Metabolic Enhancement During Initial Assessment and Treatment in Emergency care; MI: Myocardial infarction; STEMI: ST-segment elevation myocardial infarction.

\section{Competing interests}

The authors declare that they have no competing interest.

\section{Authors' contribution}

All authors contributed extensively to the work presented in this paper. Dr. Harry Selker and Joni Beshansky ran the IMMEDIATE Trial and the biomarker 
analysis for this project, as well as guided in the interpretation of data analysis, and writing of the manuscript. Dr. Gordon Huggins supervised the project and provided guidance on the interpretation of data analysis and writing of the manuscript. Robin Ruthazer contributed extensively to the analysis of the data, interpretation of results and writing of the manuscript. Dr. Inga Peter, Dr. Madhab Ray, and Dr. Jayanta T Mukherjee helped in interpretation of results and manuscript writing. Hadeel Alkofide ran the analysis and wrote the manuscript. All authors read and approved the final manuscript.

\section{Acknowledgements}

The IMMEDIATE Trial was funded by the National Institutes of Health cooperative agreement from National Heart, Lung and Blood Institute (U01HL077821, U01HL077826, U01HL077823). The IMMEDIATE Trial is registered at www.ClinicalTrials.gov (NCT00091507). The project described was supported by the National Center for Advancing Translational Sciences, National Institutes of Health (Grant Numbers UL1 TR000073 and UL1 TR001064). The content is solely the responsibility of the authors and does not necessarily represent the official views of the $\mathrm{NIH}$. Hadeel Alkofide is supported by a scholarship from King Saud University, Riyadh, Saudi Arabia.

\section{Author details}

${ }^{1}$ Clinical and Translational Science Graduate Program, Sackler School of Biomedical Sciences, Tufts Clinical and Translational Science Institute, Tufts University, Boston, MA, USA. ${ }^{2}$ Department of Clinical Pharmacy, College of Pharmacy, King Saud University, Riyadh, Saudi Arabia. ${ }^{3}$ MCRI Center for Translational Genomics, Molecular Cardiology Research Institute, Tufts Medical Center, Boston, MA, USA. ${ }^{4}$ Center for Cardiovascular Health Services Research, Institute for Clinical Research and Health Policy Studies, Tufts Medical Center, Boston, MA, USA. ${ }^{5}$ Regulatory and Clinical Research Management, Department of Health Sciences, Regis College, Weston, MA, USA. ${ }^{6}$ Tufts Clinical and Translational Science Institute, Tufts University, Boston, MA, USA. 'D Department of Genetics and Genomic Sciences, Icahn School of Medicine at Mount Sinai, New York, NY, USA.

\section{Received: 16 January 2015 Accepted: 19 November 2015 Published online: 03 December 2015}

\section{References}

1. Lee L, Horowitz J, Frenneaux M. Metabolic manipulation in ischaemic heart disease, a novel approach to treatment. Eur Heart J. 2004;25(8):634-41. doi:10.1016/j.ehj.2004.02.018.

2. Hadj A, Pepe S, Marasco S, Rosenfeldt F. The principles of metabolic therapy for heart disease. Heart Lung Circ. 2003;12 Suppl 2:S55-62.

3. Schofield RS, Hill JA. Role of metabolically active drugs in the management of ischemic heart disease. Am J Cardiovasc Drugs. 2001;1(1):23-35.

4. Hadj A, Pepe S, Rosenfeldt F. The clinical application of metabolic therapy for cardiovascular disease. Heart Lung Circ. 2007;16 Suppl 3:S56-64. doi:10.1016/j.hlc.2007.04.001.

5. Sodi-Pallares D, Testelli MR, Fishleder BL, Bisteni A, Medrano GA, Friedland C, et al. Effects of an intravenous infusion of a potassium-glucose-insulin solution on the electrocardiographic signs of myocardial infarction. A preliminary clinical report. Am J Cardiol. 1962;9:166-81.

6. Apstein CS, Opie LH. Glucose-insulin-potassium (GIK) for acute myocardial infarction: a negative study with a positive value. Cardiovasc Drugs Ther. 1999;13(3):185-9.

7. Opie LH, Bruyneel K, Owen P. Effects of glucose, insulin and potassium infusion on tissue metabolic changes within first hour of myocardial infarction in the baboon. Circulation. 1975;52(1):49-57.

8. Malmberg K, Ryden L, Efendic S, Herlitz J, Nicol P, Waldenstrom A, et al. Randomized trial of insulin-glucose infusion followed by subcutaneous insulin treatment in diabetic patients with acute myocardial infarction (DIGAMI study): effects on mortality at 1 year. J Am Coll Cardiol. 1995;26(1):57-65.

9. Mehta SR, Yusuf S, Diaz R, Zhu J, Pais P, Xavier D, et al. Effect of glucoseinsulin-potassium infusion on mortality in patients with acute ST-segment elevation myocardial infarction: the CREATE-ECLA randomized controlled trial. JAMA. 2005;293(4):437-46. doi:10.1001/jama.293.4.437.

10. Timmer JR, Svilaas T, Ottervanger JP, Henriques JP, Dambrink JH, van den Broek SA, et al. Glucose-insulin-potassium infusion in patients with acute myocardial infarction without signs of heart failure: the Glucose-Insulin-
Potassium Study (GIPS)-II. J Am Coll Cardiol. 2006;47(8):1730-1. doi:10.1016/j.jacc.2006.01.040.

11. Oliver MF, Opie LH. Effects of glucose and fatty acids on myocardial ischaemia and arrhythmias. Lancet. 1994;343(8890):155-8.

12. Malmberg K. Prospective randomised study of intensive insulin treatment on long term survival after acute myocardial infarction in patients with diabetes mellitus. DIGAMI (Diabetes Mellitus, Insulin Glucose Infusion in Acute Myocardial Infarction) Study Group. BMJ. 1997;314(7093):1512-5.

13. Apstein CS. Glucose-insulin-potassium for acute myocardial infarction: remarkable results from a new prospective, randomized trial. Circulation. 1998;98(21):2223-6.

14. van der Horst IC, Zijlstra F. van 't Hof AW, Doggen CJ, de Boer MJ, Suryapranata $\mathrm{H}$ et al. Glucose-insulin-potassium infusion inpatients treated with primary angioplasty for acute myocardial infarction: the glucose-insulin-potassium study: a randomized trial. J Am Coll Cardiol. 2003;42(5):784-91.

15. Selker HP, Beshansky JR, Sheehan PR, Massaro JM, Griffith JL, D'Agostino RB, et al. Out-of-hospital administration of intravenous glucose-insulinpotassium in patients with suspected acute coronary syndromes: the IMMEDIATE randomized controlled trial. JAMA. 2012;307(18):1925-33. doi:10.1001/jama.2012.426.

16. Grossman AN, Opie LH, Beshansky JR, Ingwall JS, Rackley CE, Selker HP. Glucose-insulin-potassium revived: current status in acute coronary syndromes and the energy-depleted heart. Circulation. 2013;127(9):1040-8. doi:10.1161/CIRCULATIONAHA.112.130625.

17. Addo TA, Keeley EC, Cigarroa JE, Lange RA, de Lemos JA, Dobbins RL, et al. Effect of glucose-insulin-potassium infusion on plasma free fatty acid concentrations in patients undergoing primary percutaneous coronary intervention for ST-elevation myocardial infarction. Am J Cardiol. 2004; 94(10):1288-9. doi:10.1016/j.amjcard.2004.07.115.

18. Chaudhuri A, Janicke D, Wilson MF, Tripathy D, Garg R, Bandyopadhyay A, et al. Anti-inflammatory and profibrinolytic effect of insulin in acute STsegment-elevation myocardial infarction. Circulation. 2004;109(7):849-54. doi:10.1161/01.CIR.0000116762.77804.FC.

19. Spagnoli LG, Bonanno E, Sangiorgi G, Mauriello A. Role of inflammation in atherosclerosis. J Nucl Med. 2007;48(11):1800-15. doi:10.2967/jnumed.107.038661.

20. Sano T, Tanaka A, Namba M, Nishibori Y, Nishida Y, Kawarabayashi T, et al. Creactive protein and lesion morphology in patients with acute myocardial infarction. Circulation. 2003;108(3):282-5. doi:10.1161/01.CIR.0000079173.84669.4F.

21. Pietila K, Harmoinen A, Hermens W, Simoons ML, Van de Werf F, Verstraete M. Serum C-reactive protein and infarct size in myocardial infarct patients with a closed versus an open infarct-related coronary artery after thrombolytic therapy. Eur Heart J. 1993;14(7):915-9.

22. Anzai T, Yoshikawa T, Shiraki H, Asakura Y, Akaishi M, Mitamura H, et al. Creactive protein as a predictor of infarct expansion and cardiac rupture after a first Q-wave acute myocardial infarction. Circulation. 1997;96(3):778-84.

23. Pietila KO, Harmoinen AP, Jokiniitty J, Pasternack Al. Serum C-reactive protein concentration in acute myocardial infarction and its relationship to mortality during 24 months of follow-up in patients under thrombolytic treatment. Eur Heart J. 1996;17(9):1345-9.

24. Andreotti F, Hackett DR, Haider AW, Roncaglioni MC, Davies GJ, Beacham JL, et al. Von Willebrand factor, plasminogen activator inhibitor-1 and Creactive protein are markers of thrombolytic efficacy in acute myocardial infarction. Thromb Haemost. 1992;68(6):678-82.

25. Armstrong EJ, Morrow DA, Sabatine MS. Inflammatory biomarkers in acute coronary syndromes: part II: acute-phase reactants and biomarkers of endothelial cell activation. Circulation. 2006;113(7):e152-5. doi:10.1161/ CIRCULATIONAHA.105.595538.

26. Parikh SV, Abdullah SM, Keeley EC, Cigarroa JE, Addo TA, Warner JJ, et al. Effect of glucose-insulin-potassium (GIK) infusion on biomarkers of cardiovascular risk in ST elevation myocardial infarction (STEMI): insight into the failure of GIK. Diab Vasc Dis Res. 2007;4(3):222-5. doi:10.3132/dvdr.2007.043.

27. Hashemian MVA, Akaberi A. Effect of glucose-insulin-potassium on Plasma concentrations of C-reactive protein in acute ST- Elevation Myocardial Infarction; A Randomized Clinical Trial. Pak J Med Sci. 2011;27(3):4.

28. Hombach V, Grebe O, Merkle N, Waldenmaier S, Hoher M, Kochs M, et al. Sequelae of acute myocardial infarction regarding cardiac structure and function and their prognostic significance as assessed by magnetic resonance imaging. Eur Heart J. 2005;26(6):549-57. doi:10.1093/eurheartj/ ehi147. 
29. Lagrand WK, Visser CA, Hermens WT, Niessen HW, Verheugt FW, Wolbink GJ, et al. C-reactive protein as a cardiovascular risk factor: more than an epiphenomenon? Circulation. 1999;100(1):96-102.

30. Suleiman M, Khatib R, Agmon Y, Mahamid R, Boulos M, Kapeliovich M, et al. Early inflammation and risk of long-term development of heart failure and mortality in survivors of acute myocardial infarction predictive role of C-reactive protein. J Am Coll Cardiol. 2006;47(5):962-8. doi:10.1016/j.jacc.2005.10.055.

31. Ohlmann P, Jaquemin L, Morel O, El Behlgiti R, Faure A, Michotey MO, et al. Prognostic value of $\mathrm{C}$-reactive protein and cardiac troponin I in primary percutaneous interventions for ST-elevation myocardial infarction. Am Heart J. 2006;152(6):1161-7. doi:10.1016/j.ahj.2006.07.016.

32. Haase J, Bayar R, Hackenbroch M, Storger H, Hofmann M, Schwarz CE, et al. Relationship between size of myocardial infarctions assessed by delayed contrast-enhanced $\mathrm{MRI}$ after primary $\mathrm{PCl}$, biochemical markers, and time to intervention. J Interv Cardiol. 2004;17(6):367-73. doi:10.1111/j.1540-8183. 2004.04078.x.

33. Orn S, Manhenke C, Ueland T, Damas JK, Mollnes TE, Edvardsen T, et al. Creactive protein, infarct size, microvascular obstruction, and left-ventricular remodelling following acute myocardial infarction. Eur Heart J. 2009;30(10): 1180-6. doi:10.1093/eurheartj/ehp070.

34. Gruzdeva O, Uchasova E, Dyleva Y, Belik E, Shurygina E, Barbarash O. Insulin resistance and inflammation markers in myocardial infarction. J Inflamm Res. 2013;6:83-90. doi:10.2147/JIR.S43081.

35. Ridker PM, Rifai N, Stampfer MJ, Hennekens CH. Plasma concentration of interleukin-6 and the risk of future myocardial infarction among apparently healthy men. Circulation. 2000;101(15):1767-72.

\section{Submit your next manuscript to BioMed Central and we will help you at every step:}

- We accept pre-submission inquiries

- Our selector tool helps you to find the most relevant journal

- We provide round the clock customer support

- Convenient online submission

- Thorough peer review

- Inclusion in PubMed and all major indexing services

- Maximum visibility for your research 